\title{
Das Verhältnis von Innen- und Außenpolitik in Entwicklungslän- dern: Der Fall Mexiko
}

\author{
von Günther Maihold
}

Die Verschuldungskrise sowie die zu ihrer Beherrschung von internationalen Organisationen vorgeschlagenen und durchgesetzten Programme außenwirtschaftlicher und wirtschaftspolitischer Art haben die Frage nach Möglichkeiten und Grenzen der innenpolitischen Handlungsfähigkeit in Entwicklungsländern verstärkt in die Diskussion gebracht. IWF und Weltbank verfolgen mit ihren vor allem ökonomisch ausgerichteten Strategien eine Politik, die die Internationalisierung der bisher national kontrollierten Sektoren der Produktionsstruktur und die Priorität von Effizienzkriterien über sozialpolitisch begründete nationale Maßnahmen zur Folge hat. ${ }^{1}$ In nur geringem Maße gehen in die Programme dieser internationalen Organisationen politische Kalküle ein, wie sie etwa in der Unterstützung der demokratischen Zukunft Argentiniens zum Ausdruck kamen. So haben Fragen des außenwirtschaftlichen Gleichgewichts Vorrang vor den Problemen der Innenpolitik, denen sich die Regime in Entwicklungsländern zu stellen haben. Für diese politischen Führungen wird die Gestaltung der innerstaatlichen Einrichtungen und Verhältnisse angesichts der Einschränkung ihres ohnehin geringen ökonomischen Potentials und ihrer innenpolitischen Handlungskompetenz zu einem Drahtseilakt, da die von ihnen mitzutragenden Maßnahmepakete die Legitimität und Stabilität der politischen Herrschaft erschüttern müssen. Insbesondere demokratisch orientierte Regime versuchen daher durch die symbolische Politik hinhaltenden Widerstandes und radikaler Rhetorik die Gefährdung ihrer Macht so gering wie möglich zu halten.

Eine Untersuchung dieses Spannungsverhältnisses von außen(wirtschafts)politischen Notwendigkeiten und innenpolitischen Herrschaftsmaximen knüpft an klassische Bestandteile der Lehre von den internationalen Beziehungen an, die in der Diskussion um den Primat der Außenpolitik bzw. Innenpolitik deutlich wurden. Im folgenden sollen die Ergebnisse dieser Úberlegungen anhand der Literatur über den außenpolitischen Prozeß in Entwicklungsländern überprüft werden und auf den mexikanischen Fall Anwendung finden.

1 Vgl. Roberto Fraenkel/Guillermo O'Donnell: The 'Stabilization Programs` of the International Monetary Fund and Their Internal Impacts, in: Richard R. Fagen (ed.): Capitalism and the State in U.S.-Latin American Relations, Stanford 1979, S. 171-216. 


\section{Der analytische Wert der Primatsdiskussion}

Die Überzeugung von der Eigengesetzlichkeit der Außenpolitik als dem Kerngehalt der Staatsräson älterer Form und der nationalen Selbstverwirklichung neuerer Prägung stellt das Grundelement jener normativ und historisch orientierten Ansätze dar, die den Territorialstaat wegen seiner als undurchdringbar angesehenen Schale ${ }^{2}$ zum einzigen Akteur der internationalen Politik erheben. Dies erlaubt natürlich eine eindeutige Unterscheidung von Innen- und Außenpolitik, die jedoch mit der Erosion der klassischen Grundlage des Nationalstaates - der Souveränität - verschwindet. Die Mehrzahl der Autoren legt heute daher weniger Wert auf die Charakterisierung der Akteure in der internationalen Politik anhand der rechtlichen Qualität, sondern bevorzugt das Verhaltensattribut der Autonomie. ${ }^{3}$ Autonome politische Systeme zeichnen sich vor allem dadurch aus, daß die Reaktion der Einheiten auf äußere Einflüsse selbst bei gründlicher Kenntnis der Beschaffenheit ihrer Handlungsumwelt nicht voraussagbar ist. ${ }^{4}$ In sinnvoller Weise kann dieser Autonomiebegriff aber nur durch die Einbettung in das Konzept des Handlungsspielraumes verwendet werden; dieses gestattet es, die Kapazitäten und Grenzen autonomer Handlungsweisen zu bestimmen.

Insoweit die Entwicklungsländer aufgrund ihrer vielgestaltigen Abhängigkeiten als 'penetrierte Systeme` ${ }^{5}$ begriffen werden können, werden für ihr Handeln objektive Grenzen gesehen, die sich aus den Kapazitäten der betreffenden Gesellschaft, deren Stellung in der Hierarchie des internationalen Systems und dessen Charakter ergeben. Diese Grenzen sind jedoch nicht konstant, der Handlungsspielraum ${ }^{6}$ kann grundsätzlich ausgeweitet, verschwendet (wenn er nicht genutzt wird) und wieder eingeengt werden (wenn er überzogen wird).

Durch die Penetration von Innen- und Außenpolitik hat sich die Primatsfrage nunmehr auf das gemeinsame Motiv innen- und außenpolitischer Interessen konzentriert, den Primat der Systemerhaltung. ${ }^{7}$ Auf dieser Basis lassen sich diese beiden Seiten staatlicher Gesamtpolitik nunmehr in der Wahl der Methoden und in den Objekten ihres Handelns unterscheiden. Eine Konkretisierung in dieser Hinsicht stellt der Versuch dar, den Zusammenhang der Ziele innen- und außenpolitischen Handelns entlang der Dimension von Freiheit und Notwendigkeit zu definieren. ${ }^{8}$ In dieser Zieltypologie lassen sich drei

2 Vgl. John H. Herz: Weltpolitik im Atomzeitalter, Stuttgart 1961, S. 26 ff. und Arnold Wolfers: Discord and Collaboration, Baltimore 1971, S. $19 \mathrm{ff}$.

3 Vgl. Richard W. Mansbach/Yale H. Fergueson/Donald A. Lampert: The Web of World Politics. Nonstate Actors in the Global System, Engelwood Cliffs 1976, S. 3 ff.

4 Vgl. Raymond Hopkins/Richard W. Mansbach: Structure and Process in International Politics, New York 1973, S. 4 ff.

5 Vgl. hierzu: James N. Rosenau: Pre-theories and Theories of Foreign Policy, in: Barry Farrell (ed.): Approaches to Comparative and International Politics, Evanston 1966, S. 65 ff.

6 Vgl. zur Definition und als Fallstudie: Rolf Hanisch: Der Handlungsspielraum eines Landes der Peripherie im internationalen System. Der Fall Ghana, Saarbrücken 1975.

7 Vgl. Wilhelm Grewe: Spiel der Kräfte in der Weltpolitik, Düsseldorf 1970, S. 258 ff. und Ekkehard Krippendorf: Ist Außenpolitik Außenpolitik? In: Politische Vierteljahresschrift Bd. 4/1963, S. 247.

8 Vgl. hierzu und im folgenden Ernst-Otto Czempiel: Der Primat der auswärtigen Politik, in: Politische Vierteljahresschrift Bd. 4/1963, S. $267 \mathrm{ff}$. 
große Gruppen unterscheiden: Ziele, die aus Existenznotwendigkeiten folgen, sind auf Sicherheit und Nahrung gerichtet. Aus Bedürfnissen entstehende Ziele beziehen sich auf Entfaltung und Entwicklung, während Programmziele die Vergrößerung von Reichtum und/oder Herrschaft bezwecken.

Historisch gesehen läßt sich bei den existenznotwendigen Zielen eine Tendenz feststellen, die von außen nach innen verläuft, d. h. insbesondere Ernährung, aber in zunehmendem Maße auch die Sicherheit sind zu einem innenpolitisch realisierten Ziel geworden. Die durch Bedürfnisse definierten Ziele und ihre Realisierung hängen in entscheidendem Maße von der Orientierung der politischen Führungsschicht ab, die vom Aufstieg profitiert. Dem gesellschaftlichen Bereich ist in historischer Perspektive größeres Gewicht zugewachsen, die Wirtschaft wurde zum Faktor der Entwicklung, die Außenpolitik zu ihrer Funktion. Mit der Betonung des innenpolitischen Raumes verbunden ergibt sich so eine Parzellierung des Verhältnisses von Innen- und Außenpolitik, insofern Teile der Wirtschaft auch Ziele der Außenpolitik beeinflussen. Bei Bedürfniszielen ist es also denkbar, daß Innen- und Außenpolitik unterschiedlichen Kalkülen folgen und auch sich widersprechende Strategien implementieren. Programme stammen aus Entscheidungen, die den höchsten Grad menschlicher Freiheit aufweisen und daher auch hinsichtlich der Abhängigkeit von Innen- und Außenpolitik sehr inkohärent sind und nur in partikularer Untersuchung geklärt werden können.

\section{Charakteristika des außenpolitischen Prozesses in Entwicklungsländern}

Die Ableitung des Modells einer Zieltypologie aus den Erfahrungen der entwickelten Staaten könnte den Vorwurf eines ethnozentrischen Vorgehens hervorrufen; allerdings erscheint die bisher zugrundegelegte Methode so lange vertretbar, wie der westliche Staat von den führenden Politikern der Entwicklungsländer selbst als politische ZielgröBe angestrebt wird. ${ }^{9}$ Auch die Untersuchung der Wahrnehmung internationaler Politik durch die Staaten der Dritten Welt unterstreicht diese Position. ${ }^{10}$

Untersuchungen, die sich auf die Erforschung spezifischer Kennzeichen der auswärtigen Beziehungen von Entwicklungsländern beziehen, sind in der Regel an Einzelfällen orientiert. Dementsprechend steht eine umfassendere Perspektive vor dem Problem einer unzulässigen Verallgemeinerung einerseits und der den Einzelfall nicht erfassenden Allgemeinaussage andererseits.

Auch methodisch sind erhebliche Unterschiede und damit verbundene Vorteile und Gefahren nachweisbar: So werden verschiedene sissue-areası und ihre Verknüpfung mit aus-

9 Vgl. Robert C. Good: State-Building as a determinant of foreign policy in the New States, in: Lawrence W. Martin (ed.): Neutralism and Nonalignment, Westport 1962, S. 3-12 sowie H. C. F. Mansilla: Latin America within the Third World: The Search for a new Identity, the acceptance of old contents, in: Ibero-Amerikanisches Archiv Bd. 11/1985, S. 180.

10 Vgl. Juan Carlos Puig: Introducción, in: América Latina: Políticas exteriores comparadas, T. 1, Buenos Aires 1984, S. 29 ff. 
wärtiger Politik untersucht, ${ }^{11}$ anhand von typisierten Entwicklungswegen werden unterschiedliche strategische und taktische Elemente für die jeweilige Außenpolitik abgeleitet $^{12}$ und auch Differenzierungen der Entwicklungsländer nach Kriterien des Entwicklungsniveaus, territorialer Größe und geographischer Lage werden als Basis für Typen auswärtigen Verhaltens herangezogen.

Ausgangspunkt einer Analyse des außenpolitischen Verhaltens von Ländern der Dritten Welt muß die Feststellung sein, daß sie in der Regel keinen Einfluß auf den Charakter des internationalen Systems nehmen können. Sowohl das Verhältnis zwischen den kapitalistischen und sozialistischen Ländern im Rahmen des Ost-West-Konfliktes wie auch die Entwicklungstendenzen des kapitalistischen Weltmarktes entziehen sich weitgehend ihren Handlungsmöglichkeiten; insgesamt betrachtet stellt das internationale System für sie eine unveränderbare Konstante dar.

Dynamisch gesehen sind Länder auf einem relativ geringen Entwicklungsniveau von der Ausrichtung der Außenpolitik nach den Notwendigkeiten der Innenpolitik geprägt. Durch die Priorität, die der Staats- und Nationsbildung aus der Orientierung an dem westlichen Vorbild erwächst, folgt ein Uberwiegen innenpolitischer Maximen. Außenpolitik wird dabei als Verlängerung der Unabhängigkeitsbewegung, eine Art symbolischer Kontinuität des Unabhängigkeitskampfes betrieben. ${ }^{13}$ Sie soll nationale Identität und staatliche Integrität schaffen, da sie wegen ihrer geringeren Umstrittenheit besser als innenpolitische Maßnahmen geeignet ist, Legitimierungsfunktionen zu übernehmen; gleichzeitig ist es für die politische Führung leichter, nach außen jene Führungsqualitäten zu beweisen, die im binnenstaatlichen Raum nur schwer zu demonstrieren sind. ${ }^{14}$ Andererseits ermöglicht diese Sachlage der politischen Führung eine größere Variation in der Außenpolitik, da kaum eine Kontrolle durch innenpolitische oder bürokratische Kräfte stattfindet. Diese große Flexibilität ist die typische Methode der Machterhaltung einer In-group, die sich zur Abdeckung konservativer Innenpolitik auf radikales außenpolitisches Verhalten stützt. ${ }^{15}$

In dem Maße, in dem sich nach obiger Typologie die Ziele vom Pol absoluter Notwendigkeit auf die Ebene der Bedürfnisse verschieben, verliert die Außenpolitik ihren Charakter, Reflex innenpolitischer Bedingungen zu sein; es sind also nicht mehr Staatsbildung und Regimeerhaltung die entscheidenden Größen, vielmehr gewinnt das Entwicklungsinteresse an Bedeutung. Im Gefolge der Modernisierung bricht die Trennung von Innenpolitik und auswärtigen Angelegenheiten zusammen. ${ }^{16}$ Die Folgen dieser Interde-

11 Vgl. Elizabeth G. Ferris: Toward a theory for the comparative analysis of Latin American Foreign Policy, in: dies./Jennie K. Lincoln (eds.): Latin American Foreign Policies: Global and Regional Dimensions, Boulder 1980, S. 239-257.

12 Vgl. Kenneth M. Coleman/Luis Quiros-Varela: Determinants of Latin American Foreign Policies, in: Ferris/Lincoln op. cit., S. 39-59.

13 Vgl. Robert C. Good, op. cit., S. 5 f.

14 Ebenda, S. 8 f.

15 Vgl. Robert L. Rothstein: Foreign Policy and Development Policy: From Nonalignment to International Class War, in: International Affairs Vol. 52/1976, S. 602.

$16 \mathrm{Vgl}$. Edward L. Morse: Modernization and the Transformation of International Relations, New York 1976, S. 84 . 
pendenz, die sich aus der Úberlagerung von externen Bedingungen und internen Konsequenzen bzw. vice versa ergeben, werden negativ erfahren und münden in eine explizite Bevorzugung bilateraler Beziehungen. ${ }^{17}$ Dies gilt natürlich vor allem für den ökonomischen Bereich, während sicherheits- und ordnungspolitische Interessen eher im regionalen Rahmen kollektiv organisiert werden.

Diese Diversifizierung der Gegenstandsbereiche führt dazu, daß sich unterschiedliche Handlungsmaximen z. B. für ökonomische und im engeren Sinne politische Fragen ergeben. Die politischen Gegenstandsbereiche emanzipieren sich von den übrigen grenzüberschreitenden Kontakten mit der Konsequenz, daß die Außenpolitik als suplementäre Legitimationsquelle durch ihre Orientierung auf die Exekutive erhalten bleibt. Im Gegensatz zu den ökonomischen Fragestellungen geraten die politischen Außenbeziehungen nicht in dem Maße in die kontroverse innenpolitische Diskussion. ${ }^{18}$ Die Realisierung der Bedürfnisziele von Entwicklung und Entfaltung steht im Gegensatz zu den notwendigen Zielen nicht unter der beinahe ausschließlichen Verfügung der politischen Elite. Mit dem Ubergang zu Programmzielen ist insofern wiederum eine Vereinheitlichung außenpolitischen Verhaltens hergestellt als wirtschaftliche wie politische Aktionen innenpolitischer Kontroverse unterliegen. Die Legitimationskraft politischer Außenbeziehungen schwindet, die Systemstabilität und -prosperität kann nicht mehr durch die Unterordnung der Außenpolitik unter innenpolitische Maximen (wie etwa in den früheren Stadien der Entwicklung) oder die Nutzung der Diversifizierung von kontroversen ökonomischen und weniger umstrittenen politischen Beziehungen (wie in der Zeit beginnender Entwicklungsfortschritte) gesichert werden. Da auf der Ebene von Programmzielen ohnehin nicht die Konsistenz von innen- und außenpolitischen Aktionen erwartet werden kann, ist nur die Frage nach dem instrumentalen Charakter beider Politikbereiche von Interesse.

In dem Begriff der 'Umstrittenheit ' politischen Handelns wurde so das geeignete Kriterium gefunden, um eine dauerhafte Funktionalisierung der Außenpolitik für innenpolitische Zwecke zu bestreiten.

Wenn man die Wege zu den Zielen von Industrie- und Entwicklungsländern vergleicht, fällt sofort der Unterschied auf: Während die Industriestaaten historisch gesehen existenznotwendige Ziele zunächst außenpolitisch und später innenpolitisch zu erreichen suchten, liegt bei den Entwicklungsländern durch die Úbernahme des westlichen Leitbildes der Identität von Staat und Nation der Ausgangspunkt zur Annäherung an diese Ziele im innenpolitischen Bereich. Diese Priorität der Staats- und Nationsbildung hat auch Konsequenzen auf der Ebene von Bedürfnis- und Programmzielen, die im Spannungsfeld von Legitimationsnotwendigkeiten und innenpolitischen Folgen des Entwicklungsfortschritts bzw. internationaler Interdependenz ihre konkrete Ausbildung finden. Die Geschwindigkeit des Fortschreitens dieser Prozesse ergibt sich zum einen aus der

17 Vgl. John J. Stremlan: The Foreign Policies of the Developing Countries in the 1980s, in: ders. (ed.): The Foreign Policy Priorities of the Third World States, Boulder 1982, S. 3.

$18 \mathrm{Vgl}$. Rothstein, op. cit., S. 602. 
Dynamik des Entwicklungsfortschritts und zum anderen der inneren politischen Struktur des jeweiligen Landes (Autoritarismus, Korporativismus etc.).

\section{Der Fall Mexiko}

Eine Fülle von Untersuchungen hat die Außenpolitik Mexikos zum Gegenstand, so daß an dieser Stelle nur auf die für das Thema relevanten Fragestellungen eingegangen werden kann. ${ }^{19}$ Ohne die umstrittene Frage nach Konstanten und Variablen der mexikanischen Außenpolitik vollständig beantworten zu können, sollen einige Grundelemente und Rahmenbedingungen angeführt werden.

In einer vorrangig geopolitisch orientierten Analyse außenpolitischer Prozesse könnte man im Fall Mexiko von einem eindeutigen Primat der Außenpolitik sprechen; die 3000 km lange Grenze zu den USA sowie die damit verbundene historische Erfahrung militärischer Intervention und kultureller Penetration erheben die Berücksichtigung des Interesses der USA zu einer Notwendigkeit jeglichen politischen Handelns der mexikanischen Regierung. Dieser rein geographische Kontext wird ergänzt durch eine vor allem wirtschaftlich und kulturell geprägte Abhängigkeit, die sich mit der Konzentration auf einen Handelspartner, gemeinsamen soziokulturellen Orientierungsmustern sowie politischen Normgefügen beschreiben läßt. Die Distanz zwischen dem ungewünschten/gewünschten Leitbild und der mexikanischen Realität erklärt einen hohen Prozentsatz der rhetorischen Radikalität und der ungebrochenen Kontinuität des mexikanischen Nationalismus auf der Suche nach eigenständiger Identität.

Andererseits kann das Interesse der USA an der bleibenden Stabilität ihres einzigen südlichen Grenzpartners als Konstante der Mexiko-Politik Washingtons angesehen werden, ohne daß dies sofort als Entschränkung des mexikanischen Handlungsspielraumes interpretiert werden müßte. Dieser "Sicherheitsschatten ${ }^{20}$ behindert die Flexibilität der politischen Strukturen sowie den notwendigen gesellschaftlichen Wandel Mexikos und sichert so die Vorherrschaft des PRI ${ }^{21}$ ab.

Die Funktionsweise und Effizienz externer und interner linkages ${ }^{22}$ definieren so einen Handlungsrahmen, der als ungewollter Partikularismus bezeichnet werden kann. Neben diesen eher objektiven Grenzen nationaler Autonomie sind auch die subjektiven Einschränkungen zu beachten, die sich aus der Antizipation einer möglichen Berührung von Interessen der USA durch das Handeln Mexikos ergeben könnten.

$19 \mathrm{Vgl}$. die Studie von Mario Ojeda: Alcances y límites de la política exterior de México, México 1984 und den Sammelband von Olga Pellicer (ed.): La política exterior de México: Desafios en los ochenta, México 1973.

20 Vgl. Manfred Mols: Mexiko im 20. Jahrhundert, Paderborn 1981, S. 163.

21 Zur Einschätzung des PRI (Partido Revolucionario Institucional) und seiner Politik, vgl. Volker Lehr: Der mexikanische Autoritarismus, München 1981.

22 Siehe hierzu: Michael O’Leary: Linkages between domestic and international politics in underdeveloped nations, in: James N.Rosenau (ed.): Linkage Politics, New York 1969, S. 324-346 sowie mit breiterem theoretischen Zugang: Peter Gourevitch: The second image reversed: the international sources of domestic politics, in: International Organization Vol. 32/1978. 
Der Handlungsspielraum eines Landes ist auch durch seine Position in der Hierarchie des internationalen Systems bedingt; Mexikos Strategie läßt sich in dieser Hinsicht als eine Politik der Nicht-Einbindung beschreiben, die sowohl die Optionen einer Führungsrolle wie gezielter Passivität offenläßt. Während eine Fülle von Ländern der Dritten Welt außenpolitische Stärke durch Bindung an internationale Organisationen und Zusammenschlüsse steigern wollen, sieht Mexiko die Basis einer solchen Stärkung in der Fähigkeit zu flexibler Außenpolitik, die gerade durch die Einbindung nationaler Interessen in internationale Organisationen, deren Politik Mexiko dann nur marginal zu steuern vermag, ausgeschlossen würde. Dieser gewollte Partikularismus fordert die Bewahrung der nationalen Optionen der Außenpolitik in eigener Hand, seien sie auch nur formaler oder symbolischer Art.

\section{1. Außenpolitik als Verlängerung der Innenpolitik: Nicht-Intervention und Selbst- bestimmung}

Die mexikanische Außenpolitik der Postrevolution bis 1972 ist allgemein als passiv, reaktiv und essentiell negativ ${ }^{23}$ beschrieben worden, da sie sich in besonderer Weise durch eine einseitige Gründung auf völkerrechtliche Normen auszeichnet.

Diese Position läßt sich auf die internationale Ausweitung von Prinzipien und Praktiken zurückführen, die in der mexikanischen Revolution entwickelt und getestet wurden. ${ }^{24}$ Durch die Einbettung in die special relationship mit den USA waren Mexiko die Hände für eine offensive Außenpolitik gebunden, die Betonung symbolischer Tradition sowie der moralischen Überzeugung in Einzelfällen war die geeignete Strategie, der mexikanischen Entwicklung seit der Revolution internationales Ansehen zu verschaffen. Die Konzentration auf die Verwirklichung existenznotwendiger und im Bedürfnisbereich angesiedelter Entwicklungsziele bei Aufrechterhaltung störungsfreier Beziehungen zu den USA versprach einen schnellen Entwicklungsfortschritt. Mit der Betonung völkerrechtlicher Normen war gleichzeitig ein unverdächtiges - da von politischen Opportunitätserwägungen unabhängiges - Instrument gefunden, das nicht zuließ, jede außenpolitische Äußerung als Affront gegen die USA zu werten.

Die Sicherheit Mexikos ruhte so in der Stabilität der 'special relationship' sowie in der symbolischen Distanzierung von den USA durch den Rückgriff auf völkerrechtliche Normen, die gerade in der Auseinandersetzung mit dem nördlichen Nachbarn Anwendung gefunden hatten. Neben den Prinzipien der Nichteinmischung, der Selbstbestimmung und der Gleichheit aller Staaten in internationalen Organisationen sind die Betonung der Calvo-Klausel sowie die Ausarbeitung der Carranza-Doktrin, der EstradaDoktrin und der Cárdenas-Doktrin von Bedeutung.

23 Vgl. Robert E. Scott: National development and Mexico's foreign policy, in: International Journal Vol. 37/ 1981-82, S. 43.

24 Vgl. James E. Engel: The Revolution and Mexican Foreign Policy, in: Journal of Inter-American Studies and World Affairs Vol. 11/1969, S. 532. 
So faßt die Calvo-Klausel den ausdrücklichen Verzicht auf diplomatischen Schutz bei Verträgen von Ausländern mit lateinamerikanischen Regierungen ${ }^{25}$ und wird von der Carranza-Doktrin (1918) weitergeführt, die das Prinzip der Nichteinmischung und der Respektierung nationaler Unabhängigkeit festschreibt. Die politische Indifferenz mexikanischer Außenpolitik ist in der Estrada-Doktrin (1930) ausgedrückt, die die automatische Anerkennung jeweils neuer Regierungen ohne Rücksicht auf deren Legitimität enthält. Gerade die Infragestellung der Legitimität von Regierungen durch äußere Mächte als Anlaß für Interventionen sei eine Praxis, die Mexiko selbst habe bitter erfahren müssen, meinte Außenminister Estrada im Jahre 1930. Die Verankerung des Prinzips der Nicht-Einmischung in Gestalt der Estrada-Doktrin stellt einen herausragenden Beitrag der mexikanischen Außenpolitik zum Völkerrecht der neueren Zeit dar. Die CárdenasDoktrin von 1938 knüpft wiederum an die Calvo-Klausel an und weitet sie auf den Fall von staatlichen Enteignungen aus.

Diese Versuche der legalistischen Handhabung der Außenbeziehungen haben für Mexiko die Erhöhung des internationalen Prestiges gebracht; dabei spiegelt diese Politik die rationale Strategie eines schwachen Staates in der internationalen Politik wider, sich nur in differenzierten Fällen und nach eigenem Kalkül zu internationalen Fragen zu äußern. Diese selektive Partizipation an der internationalen Politik erlaubte Mexiko ökonomischen Fortschritt im Rahmen einer abhängigen Entwicklung, beinhaltete aber gleichzeitig eine isolationistische Außenpolitik, die die internationale Umwelt nur als Quelle von Gefahren wahrnimmt. ${ }^{26}$ Diese gesuchte Distanz zu den internationalen Konfliktfeldern sollte vor innen- und außenpolitischem Schaden bewahren, die Anwendung der eigenen Erfahrungen stellte die sicherste Basis für eine unkomplizierte Außenpolitik dar.

Wenn man die aus historischer Erfahrung gewachsenen Grundlagen der mexikanischen Außenpolitik betrachtet, darf ihr bis heute gültiger Grundzug der ideologischen Verpflichtung auf revolutionäre Bewegungen nicht übersehen werden. Aufgrund dieser Solidarität hat Mexiko als einziges Land der OAS die diplomatischen Beziehungen zu Kuba auch auf dem Höhepunkt der Krise 1962-1964 nicht abgebrochen und sich nicht an ökonomischen Sanktionen beteiligt. Zwar waren dafür auch innenpolitische Erwägungen maßgeblich, entscheidend ist jedoch die enge Verknüpfung der nationalen Identität des Landes mit der Revolution, so daß die Unterstützung anderer revolutionärer Regime auch ein Stück Legitimitätsbeschaffung für den innenpolitischen Führungsanspruch des PRI darstellt.

Das Signum einer 'passiven Außenpolitik wurde 1967 mit der mexikanischen Initiative für eine nuklearwaffenfreie Zone in Lateinamerika in Frage gestellt, ${ }^{27}$ die in den Vertrag von Tlatelolco mündete. So wird insbesondere die Regierungszeit des Präsidenten López

25 Vgl. hierzu: Modesto Seara Vázquez: La Política Exterior de México, México 1969, S. 5 ff.

26 Vgl. Jorge Castañeda: Revolution and Foreign Policy: Mexico's Experience, in: Carlos Alberto Astiz (ed.): Latin American International Politics, Notre Dame 1969, S. 154.

27 Vgl. Harvey J. Kaye: How 'New is Mexico's Foreign Policy? in: Interamerican Economic Affairs Vol. 28/ 1975, S. 87-92; ebenso Olga Pellicer de Brody: Veinte Años de política exterior mexicana: 1960-1980, in: Foro Internacional Vol. 21/1980, S. 153. 
Mateos (1958-1964) als Ausgangspunkt neuer Ansätze in der Außenpolitik durch regionale Kooperation, Erweiterung des außenpolitischen Handlungsspielraumes gegenüber den sozialistischen Ländern sowie die beginnende Organisation der blockfreien Staaten angesehen; letztlich ist jedoch die 'special relationship' nicht aufgegeben worden, so daß der alte Stil der Außenpolitik sich bis in die ersten Jahre der Präsidentschaft von Luis Echeverría (1970-1976) fortsetzte.

\section{2. Innenpolitik durch Außenpolitik: Diversifizierung und 'Tercermundismo}

Dieser Abschnitt mexikanischer Außenpolitik umfaßt den Zeitraum von 1972-1976, also jene Jahre, für die viele Analytiker von einer neuen Außenpolitik sprechen. Diese auch für den gesamten lateinamerikanischen Raum aufgestellte These ${ }^{28}$ sieht insofern einen Kontinuitätsbruch als neue Ziele (in unserem Sinn: Programmziele) die Ausrichtung der Außenpolitik bestimmen. Die Abwendung von der bilateralen Struktur der sozioökonomischen Beziehungen geht einher mit dem Bekenntnis zu einer aktiven Außenpolitik, die ausgeglichenere Partizipationschancen sowie einen strukturellen Wandel im System der internationalen Wirtschaftsbeziehungen vorsieht.

Seit 1972 betrachtet Mexiko die sspecial relationship mit den USA als beendet, die Diversifizierung der Außenbeziehungen wird zu einem Mittel, das die inneren Schwierigkeiten einer 'Stabilität ohne Fortschritt überwinden helfen soll. ${ }^{29}$ Einerseits waren mit dem Ende des 'milagro mexicano die Kosten einer einseitigen Exportabhängigkeit als Folge der protektionistischen Handelspolitik der USA spürbar geworden, andererseits waren die nach der politischen Krise von 1968 eingeleiteten inneren Reformmaßnahmen in den ersten Jahren der Regierungszeit Echeverrías gescheitert; ${ }^{30}$ daher läßt sich aufgrund der Chronologie sowie der Thematik der außenpolitischen Aktivitäten die These erhärten, daß die aktivistische radikale Außenpolitik des Präsidenten in großem Maße Reflex des gescheiterten internen Reformismus war. ${ }^{31}$ Die Strategie der Instrumentalisierung der Außenpolitik für innenpolitische Zwecke bot vor allem deshalb entscheidende Vorteile, da sie im Gegensatz zu einer internen Redistributionspolitik weniger umstritten und daher auch nicht mit systemgefährdenden Konfliktpotentialen behaftet war. ${ }^{32}$ Die Verfolgung einer 'diplomacía comercialı versuchte die Diversifizierung der einseitigen Abhängigkeit von den USA durch Ausbau der Beziehungen zu Kanada, Japan und Westeuropa zu erreichen, die Ubernahme einer politischen Sprecherrolle im

28 Vgl. Gerhard Drekonja: Contenidos y Metas de la Nueva Política Exterior Latinoamericana, in: Gerhard Drekonja/Juan G. Tokatlian (eds.): Teoría y Práctica de la Política Exterior Latinoamericana, Bogotá 1983, S. 22.

29 In dieser Zeit wurden zu nicht weniger als 62 Nationen diplomatische Beziehungen aufgenommen.

$30 \mathrm{Vgl}$. Yoram Shapira: La política exterior de México bajo el régimen de Echeverría: Retrospectiva, in: Foro Internacional Vol. 19/1978, S. 69.

31 Ebenda.

32 Vgl. Yoram Shapira: Mexican Foreign Policy under Echeverría, Beverly Hills 1978 (The Washington Papers Vol VI, No. 56), S. 64. 
Rahmen des Nord-Süd-Dialoges zeigt das Interesse an einer Neugestaltung der Interaktionsregeln im Rahmen der internationalen Politik. Innenpolitisch zielt diese Strategie auf die Erhöhung des Prestiges von Partei und Regierung bei der linken Intellektuellenschicht sowie auf die Ausweitung des Handlungsspielraumes gegenüber den internen linkage-Gruppen der USA im Lande ab.

Mexikos Initiative zur Sicherung rechtlicher Souveränität über Rohstoffe findet ihren Niederschlag auf multilateraler Ebene in der von den Vereinten Nationen 1974 verabschiedeten 'Charta der ökonomischen Rechte und Pflichten der Staatenı sowie - stärker ideologisch motiviert - in der bilateralen Unterstützung von Chiles Weg zum Sozialismus. Insbesondere der Besuch des chilenischen Präsidenten Allende in Mexiko sowie der Abbruch der diplomatischen Beziehungen zu Chile nach Allendes Tod $^{33}$ können die These einer externen revolutionären Legitimierung der Herrschaft des PRI und der internen Mobilisierung progressiver Kreise erhärten. ${ }^{34}$ Auch die Förderung des lateinamerikanischen Wirtschaftssystems SELA zeigt das Interesse, sowohl die Verengung auf bilaterale Bindungen aufzulösen als auch die Struktur des internationalen Wirtschaftssystems durch regionale Kooperation zu verändern.

Die Inkongruenz einer radikalen` Außenpolitik (z. B. gegenüber Franco-Spanien oder in Nord/Süd-Fragen) mit einem gemäßigten internen Reformismus und massiver staatlicher Repression hat die Instrumentalisierung der Außenpolitik zu innenpolitischen Zwecken vor allem auch in thematischer Hinsicht deutlich gemacht.

Uber das bleibende Ergebnis der Außenpolitik Echeverrías sind die Meinungen sehr divergent: Sicherlich ist zutreffend, daß diese 'neuer Außenpolitik Mexiko nicht mehr ein Rückfallen in das Ideal einer autarkieorientierten Politik ermöglicht, ${ }^{35}$ die Auswirkungen internationaler Ereignisse sind Bestandteil mexikanischer Innenpolitik. Das internationale Umfeld wird also nicht mehr nur als Gefahrenquelle begriffen, sondern als Gestaltungsbereich für nationale Interessen genutzt. Weiterhin erscheint auch der stabilisierende Einfluß der Außenpolitik auf die innenpolitische Struktur unabhängig von der inhaltlichen Einschätzung der außenpolitischen Themata unbestreitbar: "Wenn gegenüber den Linken eine Besänftigungsstrategie angewendet wurde, ist dadurch die Integration dieser Gruppen in das System gefördert und damit die Basis des Systems verbreitert worden. Wenn andererseits der Verbalradikalismus von den USA zumindest teilweise für bare Münze genommen werden mußte, hat sich dadurch die Verhandlungsposition Mexikos wenn auch nur geringfügig - verbessert. Dies kann für die Úberlebensfähigkeit des Systems nur von Nutzen sein. ${ }^{136}$

33 Die Aufkündigung der diplomatischen Beziehungen zu Chile ist ebenso wie der Abbruch der Kontakte zu Franco-Spanien 1973 als Verletzung der Estrada-Doktrin anzusehen und bietet einen weiteren Hinweis für den Strukturbruch in der mexikanischen Außenpolitik in dieser Zeit.

$34 \mathrm{Vgl}$. Maria Ojeda: Alcances y límites de la política exterior de de México, México 1984, S. 182.

$35 \mathrm{Vgl}$. Hector Manuel Ezeta: Las nuevas dimensiones de la política exterior de México, in: Pensamiento Político Vol. 21/1976, S. $200 \mathrm{f}$.

36 Wolf Grabendorff: Die innenpolitische Funktion der mexikanischen Außenpolitik, in: Lateinamerika Studien 6, München 1980, S. 557 : 


\section{3. Die Emanzipierung der Außenpolitik von der Innenpolitik}

Das unbestreitbar durch die Außenpolitik Echeverrías gewonnene Prestige des Landes im internationalen Rahmen hat für Präsident López Portillo (1976-82) die Kontinuität zum bisherigen Stil außenpolitischen Handelns nahegelegt. Zwar betonte er weniger die explizite Position des 'tercermundismo', er führte diese Sprecherrolle weiter in die internationale Politik hinein: Der Schwerpunkt lag nun auf der Ausnutzung des gewachsenen Ansehens des Landes für die Wahrnehmung einer Brückenfunktion zwischen Nord und Süd. Gefördert wurde die Ausführung dieser partikularen Rolle durch die umfangreichen Ölfunde an der karibischen Küste, die als Schlüssel zur Lösung der nationalen Probleme (vor allem die hohe Verschuldung) sowie die Umgestaltung der internationalen Handelsbeziehungen angesehen wurden. Der Schwerpunkt außenpolitischer Bemühungen lag daher in der Ausnutzung von Mexikos Verhandlungsmacht auf multilateraler Ebene: Der Vorschlag eines Weltenergieplanes 1979, die Verträge von San José 1980, die eine begünstigte Olversorgung von karibischen und zentralamerikanischen Staaten durch Mexiko und Venezuela vorsehen, sowie die Ausrichtung der als Konsequenz des Brandt-Berichtes einberufenen Gipfelkonferenz von Cancún 1981 belegen das Interesse an der Wahrnehmung einer Brückenfunktion auf multilateraler Ebene.

Dieser kollektiv und supranational angelegte Ansatz kann auch als moderne Form der traditionell legalistisch-moralischen Strategie verstanden werden, da er gleichzeitig auf eine Neuorientierung der bilateralen Beziehungen zu den USA bezogen war. ${ }^{37}$ So mochte zwar Mexikos Interesse an einer regionalen Vormachtstellung im zentralamerikanischen und karibischen Raum aus rein politischen Kalkülen des außenpolitischen Verhaltens folgen und somit in der Kontinuität der Außenpolitik Echeverrías stehen, andererseits war Mexiko paradoxerweise gerade durch das Öl - trotz größeren Verhandlungsspielraumes und gewachsenen politischen Einflusses - für amerikanischen Druck anfälliger geworden. ${ }^{38}$ Diese durch die nordamerikanischen Interessen an den Olvorkommen begründete Reorientierung im ökonomischen Bereich auf eine - wenn auch modifizierte - 'special relationship offenbart die Rückkehr zum Modell der assoziierten Entwicklung. Mexikos Anspruch auf die Rolle einer politischen Mittelmacht ${ }^{39}$ mit Brückenfunktion zeigt sich unabhängig von der neuen Orientierung an den USA im ökonomischen Bereich durch die Betonung des typischen 'gewollten Partikularismusı, einer distanzierten Politik gegenüber den kollektiven Organisationen der Dritte-Welt-Länder wie der OPEC und den Blockfreien. ${ }^{40}$ Die Suche nach einem eigenen Weg schien gerade durch die Zwischenstellung als ölbesitzendes Land der Dritten Welt gerechtfertigt und besondere Vorteile versprechend.

37 Vgl. Robert E. Scott, op. cit., S. 57.

38 Vgl. Wolf Grabendorff: Mexikos selbstbewußte Außenpolitik, in: Europa-Archiv Bd. 34/1979, S. 695.

$39 \mathrm{Vgl}$. Guadalupe González G.: Incertidumbres de una potencia media regional: Las nuevas dimensiones de la política exterior mexicana, in: Olga Pellicer (ed.): La política exterior de México: Desafíos en los ochenta, México 1973, S. 15-81. 
Das Drängen der USA auf eine neu gestaltete sspecial relationship ' in Gestalt der Idee eines Nordamerikanischen Gemeinsamen Marktes von Mexiko, Kanada und den USA oder der präferentiellen Versorgung mit Ol und $\mathrm{Gas}^{41}$ bis hin zur Propagierung des letztlich aufgeschobenen Beitritts zum GATT ${ }^{42}$ zeigt die ökonomische Dimension der Außen beziehungen Mexikos auf, das sich nun plötzlich selbst in der Lage sah, mit hinreichender Machtfülle ausgestattet über eine gleichberechtigte sspecial relationship` zu verhandeln.

Die revolutionäre Solidarität Mexikos mit Nicaragua während der Regierungszeit von López Portillo und seine den Absichten der USA weithin gegensätzliche Politik in Mittelamerika ${ }^{43}$ spiegeln die politisch motivierten Elemente der Außenpolitik wider. Mit dem Scheitern der Pläne zur Neugestaltung der Nord/Süd-Beziehungen in globalem Maßstab, die Mexiko aufgrund seiner Mittlerfunktion als Olmacht mit Dritte-WeltOrientierung in besonderem M̀aße glaubte fördern zu können, war die stärkere regionale Orientierung der politischen Außenbeziehungen vorgezeichnet.

\section{4. Die Krise und die außenpolitischen Zwänge der Innenpolitik}

Die Regierungszeit von López Portillo war dadurch gekennzeichnet, daß das Öl als innen- und außenpolitisch einsetzbares Mittel verwendet wurde; diese 'petrolización ‘ der Politik verknüpf te "unverantwortbar einseitig die Außenpolitik, die nationalen Entwicklungspläne und insbesondere das Verhältnis zu den USA mit der Olfrage ". ${ }^{44}$ Die Krise des internationalen Olmarktes mit der beginnenden Olflut mußte Mexiko so in einer kumulierten Wirkung treffen: Nachfragerückgang, Liquiditätsprobleme, Devisenmangel und hohe Auslandsverschuldung.

Diese Problemfülle brachte für das Verhältnis von Innen- und Außenpolitik eine Umkehrung des traditionellen Musters mit sich: Als Ergebnis der Außenpolitik Echeverrías und López Portillos war es zu einer Trennung von außenwirtschaftlichen und in engerem Sinne außenpolitischen Beziehungen gekommen, unterschiedliche Strategien bestimmten die Verwirklichung der Programmziele. Mit der Aktivierung der Außenpolitik war eine so starke Einbindung in die internationale Politik erfolgt, daß ein Rückzug in die frühere isolationistische Position nicht mehr möglich war. Äußere Ereignisse bestimmten nun in großem Maße die Handlungsalternativen, die der Regierung noch zur Verfügung standen. Die ökonomische Krise grenzte die innenpolitische Manövriermasse für

40 Vgl. Olga Pellicer de Brody: Veinte Años, op. cit., S. 158.

$41 \mathrm{Vgl}$. Susan Kaufman Purcell: Mexico-U.S.Relations: Big Initiatives can cause Big Problems, in: Foreign Affairs Vol. 60/1981-82, S. $383 \mathrm{ff}$.

42 Vgl. Dale Story: Trade Politics in the Third World: a case study of the Mexican GATT decision, in: International Organization Vol. 36/1982, S. 767-794.

43 Vgl. Susan Kaufman Purcell, op. cit., S. 388 f.

44 Manfred Mols/Ruth Zimmerling: Die Außenpolitik Argentiniens, Brasiliens, Mexikos und Venezuelas, in: Karl Kaiser/Hans-Peter Schwarz (Hrsg.): Weltpolitik. Strukturen-Akteure-Perspektiven, Bonn 1985, S. 688. 
die politische Führung weiter ein, das Sanierungsprogramm des Internationalen Währungsfonds verlangte Ausgabenkürzungen und Haushaltskonsolidierung, die zu Einschnitten in der Sozialpolitik und zur Entstaatlichung der Wirtschaft führten. Damit wurden die Grundlagen der postrevolutionären Außenpolitik - Nicht-Intervention und Selbstbestimmung - berührt, da mit der Privatisierung der Industrie auch der Verlust der nationalen Kontrolle über die Wirtschaft drohte.

Die zur Krisenbewältigung eingesetzten Maßnahmen belegen so den Versuch der politischen Führung unter Miguel de la Madrid, neue ökonomische Handlungsfähigkeit über die verstärkte Anlehnung an die USA (in Gestalt einer musterhaften Erfüllung des Sanierungsprogrammes) zu gewinnen. Sieht man von dem eher innenpolitisch motivierten symbolischen Schritt der Nationalisierung der Banken durch López Portillo 1982 ab, so hat sich Mexiko in den Verhandlungen mit dem IWF vergleichsweise nachgiebig das Austeritätsprogramm verordnen lassen. Der Versuch des argentinischen Präsidenten Alfonsin, die Gemeinschaft der Schuldnerländer Lateinamerikas auf multilateraler Ebene zu vereinen, stieß von seiten Mexikos auf geringe Unterstützung. Präsident de la Madrid betonte die Ablehnung rein defensiver Aktionen und erhoffte sich in bilateralen Verhandlungen einen schnelleren Ausweg aus der Schuldenkrise. ${ }^{45}$ Dieses Verfahren sollte auch das internationale Prestige Mexikos erhöhen, das durch die Nationalisierung der Banken in Finanzkreisen erheblich gelitten hatte. Erst nach den Verhandlungen mit dem IWF im September 1985 zeigte sich der Präsident einer Front der Schuldnerländer geneigt, die sich an dem vom peruanischen Präsidenten Alan García vorgeschlagenen Modell der Begrenzung des Schuldendienstes auf $10 \%$ der Exporterlöse orientiert.

Auch in seinen politischen Strategien zur Bewältigung der akuten Krise von 1982 zeigt sich wiederum die starke Tendenz der mexikanischen Politik partikulare Lösungsmodelle zu verfolgen und die Zukunft des Landes im "Sicherheitsschatten" der USA mit der Reorientierung auf eine sspecial relationship im ökonomischen Bereich zu suchen.

Auch die Zentralamerikapolitik ${ }^{46}$ hat unter dem angeschlagenen Prestige des Landes gelitten: Die Betonung der nationalen Sicherheit ${ }^{47}$ und die Vermeidung eines bewußt 'progressiven 'Auftretens in der Frage Zentralamerikas sind Versuche, die die internen Konsequenzen außenpolitischer Entscheidungen gering zu halten. So ist Miguel de la Madrid zu Nicaragua auf stärkere Distanz gegangen ${ }^{48}$ und auch die Zurückhaltung hinsichtlich Guatemalas soll die Anziehung weiterer Flüchtlinge vermeiden helfen.

Die unter López Portillos Wirtschaftspolitik aus dem nationalen Konsens herausgedrängten Mittelklassen, deren Existenzgrundlage durch hohe Inflationsraten, Abwertun-

45 Vgl. Miguel de la Madrid H.: Mexico: The New Challenges, in: Foreign Affairs Vol. 63/1984, S. 67.

46 Vgl. Edward J. Williams: Mexico's Central American Policy: Revolutionary and Prudential Dimensions, in: Michael Erisman/John D. Martz (eds.): Colossus Challenged: The Struggle for Carribbean Influence, Boulder 1982, S. 149-169.

47 Vgl. Miguel de la Madrid H., op. cit., S. 75.

48 Dem widerspricht auch nicht die Ubernahme der Lieferquoten Venezuelas an Nicaragua durch Mexiko, da Venezuela dies aus politischen Gründen abgelehnt hatte; vgl. George W. Grayson: Oil and Politics in Mexico, in: Current History Vol. 82/1983, S. 419 f. 
gen und die Nationalisierung der Banken in hohem Maße gefährdet war, ${ }^{49}$ will Präsident Miguel de la Madrid wieder an den Staat binden und ihre dauerhafte Abwanderung zum PAN verhindern.

Die regionale Ausrichtung der mexikanischen Außenpolitik im Rahmen der ContadoraGruppe $^{50}$ war die einzige politische Quelle eines steigenden internationalen Prestiges des Landes. Neben dem Interesse an der Bewahrung der nationalen Sicherheit und dem Anspruch auf regionale Führerschaft sind diese außenpolitischen Aktivitäten von den zentralen Elementen der eigenen revolutionären Erfahrung bestimmt: Selbstbestimmung und Nicht-Intervention. Der Erfolg der Contadora-Gruppe liegt für Mexiko schon allein in der Tatsache, den USA ein direktes Eingreifen in Zentralamerika erschwert und so zur Beruhigung der Lage beigetragen zu haben.

Diese im engeren Sinne politisch motivierten Außenbeziehungen sind von der außenwirtschaftlich begründeten Krise und deren Rückwirkungen auf die Innenpolitik weithin unberührt geblieben, sie haben vielmehr den nötigen Ausgleich zur außenwirtschaftlichen Handlungsunfähigkeit geschaffen. Die multilaterale Zusammenarbeit im Rahmen der Contadora-Gruppe verfolgt dabei teilweise antagonistische Strategien zu denen der USA ${ }^{51}$ während die bilaterale Beziehungsstruktur im ökonomischen Bereich ungeachtet mancher gegenteiliger Rhetorik von einer Pflege der Nachbarschaft gekennzeichnet ist. Dies zeigt sich beispielsweise in der erneuten Diskussion um einen Beitritt des Landes zum GATT, ein Schritt, an dem den USA in besonderem Maße gelegen ist.

\section{Außenwirtschaf tliche Begrenzungen und innenpolitischer Handlungsspielraum}

Wenn man die Neuordnung der Außenpolitik auf das zugrundeliegende Modell bezieht, so wird deutlich, daß die Differenzierung von ökonomischen und politischen Außenbeziehungen erhalten geblieben ist, in beiden Bereichen werden sich widersprechende Strategien verfolgt. Die Uberlagerung von existenznotwendigen Zielen, Bedürfnis- und Programmkomplexen als Handlungsmaxime hat ein einheitliches Beziehungsmuster im Verhältnis von Innen- und Außenpolitik nicht entstehen lassen, die diversifizierte Struktur läßt sich nurmehr sektoriell erfassen.

Der Einfluß äußerer Bedingungen auf die innenpolitische Krisenbewältigung in Gestalt der neuen Orientierung an den USA hat den geringen Handlungsspielraum Mexikos auf internationaler Ebene gezeitigt. Der Ausbau des außenpolitischen Potentials durch den Rückgriff auf das Öl hat sich als Bumerang erwiesen, die Konsequenz war die Einschränkung außenpolitischer Handlungsmöglichkeiten und die ökonomisch notwendige

49 Vgl. Soledad Loaeza: Las clases medias mexicanas y la coyuntura económica actual, in: Pablo González Casanova/Héctor Aguilar Camín (coord.): México ante la crisis. El impacto social y cultural/las alternativas, México 1985, S. 221-237.

$50 \mathrm{Vgl}$. den Uberblick bei René Herrera Zúniga/Manuel Chavarría: México en Contadora: Una búsqueda de límites a su compromiso en Centroamérica, in: Foro Internacional Vol. 24/1984, S. 458-483.

51 Vgl. Susan Kaufman Purcell, op. cit., S. 390. 
Anlehnung an die USA. Die Úberdehnung des Spielraumes, die aus der falschen Einschätzung des eigenen inneren Potentials sowie dem Verlust des internationalen Prestiges durch Aktionen wie die politisch und ökonomisch wenig einträgliche Nationalisierung der Banken folgte, kann nur aufgefangen werden im Rahmen einer erfolgreichen multilateralen Außenpolitik im regionalen Rahmen. Dieser Weg ermöglicht den Aufbau eines auch innenpolitisch notwendigen Gegengewichtes zur bilateralen Bindung an die USA. Allerdings können die sozialen Kosten der ökonomischen Krise vom Juni 1986 (Kursverfall des Peso, massiver Inflationsschub und akute Gefahr der externen Zahlungsunfähigkeit) dauerhaft nicht nur symbolisch aufgefangen werden, auch die politischen Strukturen werden trotz des 'Sicherheitsschattens' der USA unter Druck geraten und so zur Veränderung des Verhältnisses von Innen- und Außenpolitik beitragen. ${ }^{52}$

52 Im Vorfeld des 'destaper (der Benennung des nächsten Präsidentschaftskandidaten des PRI) ist jüngst die Demokratisierung der Partei vom ehemaligen Erziehungsminister Porfirio Muñoz Ledo und dem früheren Gouverneur des Staates Michoacán Cuauhtémoc Cárdenas gefordert worden. Im intellektuellen Umfeld der Zeitschrift 'Vuelta' wurde dies schon vorher unter dem Motto "por una democracia sin adjetivos" verlangt. Vgl. dazu Enrique Krauze: Por una democracia sin adjetivos, México 1986. 
The Relationship of Domestic and Foreign Policies in Developing Countries: The Case of Mexico

\section{By Günther Maihold}

The Stabilization Programmes of the International Monetary Fund have raised the question of the capacity of developing countries' political systems to implement the economic and socio-political programmes imposed by this international organisation. Beginning with the classical discussion of the primacy of domestic or foreign policy, an attempt is made to describe the connection between the two by means of a goal typology. Goals essential to existence, goals based on needs and programmes establish, in varying degrees of intensity, the connection between domestic and foreign policy. Elements characteristic of the foreign-policy process in developing countries are combined with the goal typology and applied to the case of Mexico.

Since the revolution of 1910 a legalistic approach to foreign policy has predominated which was based on the internal revolutionary experience that led to a selective participation in international politics. However beginning with the presidency of Echeverría, foreign policy was actively used to further domestic political goals. Based on the country's oil reserves, President López Portillo attempted to establish Mexico as a bridge between North and South in international politics. This attempt failed and led, along with the economic crisis of 1982 , to a new spezial relationship with the United States. Regional political involvement with the Contadora Group and utilisation of the slight potential in domestic policy created by the conditions imposed by the IMF characterise the relationship between domestic and foreign policy today. 